\title{
P13 Role of Adipose Tissue and Skeletal Muscle in Peripheral Arterial Disease
}

Joana Ferreira ${ }^{1, *}$, Pedro Cunha ${ }^{2}$, Alexandre Carneiro ${ }^{3}$, Cristina Cunha ${ }^{2}$, Cristina Silva ${ }^{2}$, Isabel Vila ${ }^{2}$, Amílcar Mesquita ${ }^{4}$, Jorge Cotter ${ }^{4}$

${ }^{1}$ Hospital da Senhora da Oliveira-Guimaraes

${ }^{2}$ Hospital da Senhora da Oliveira

${ }^{3}$ Centro Hospitalar de Trás-os-Montes e Alto Douro

${ }^{4}$ Hospital da Senhora da Oliveira, Guimarães, Portugal

\section{ABSTRACT}

Background: Peripheral arterial disease (PAD) is a manifestation of atherosclerosis. Adipose tissue (AT) and skeletal muscle (SM) are endocrine organs with vascular effects. The objective is to compare the quantity and function of AT and SM between two groups: intermittent claudication (IC) and critical limb ischemia (CLI).

Methods: A prospective observational study is being performed. A transverse CT image at lower border the third lumbar vertebra was used to quantify the SM, visceral and subcutaneous AT. The SM function was inferred with the determination of hand strength using a Jamar ${ }^{\circledR}$ hydraulic hand dynamometer.

Results: From December 2018 to May 2019, 44 PAD were studied: 31 with IC ( 24 male; age: 67 10, 29 years) and 13 CLI (7 male; age: 70, 62 6, 74 years). CLI have a lower quantity of subcutaneous AT and higher quantity of visceral and total AT, determined on the CT scan (subcutaneous AT: 16564,75 7600,00 $\mathrm{cm}^{2}$ IC versus 16067,42 10187,33 $\mathrm{cm}^{2}$ CLI $p=0.03$; visceral AT: 17212,20 10096, $30 \mathrm{~cm}^{2}$ IC versus 18904,85 10189,04 cm $\mathrm{cLI}^{2}$ C = 0,03; Total AT: $33222,0815459,33 \mathrm{~cm}^{2}$ IC versus $57320,0052538,78 \mathrm{~cm}^{2}$ CLI $p=0.03$ ). CLI had lower SM strength and density (muscle strength right hand: $22,628,20 \mathrm{Kgf}$ IC versus 16,38 7,84 Kgf CLI $p=0,02$; SM strength left hand: $21,988,98 \mathrm{Kgf}$ IC versus 16,37 8,35 Kgf CLI $p=0,04$; SM density: 20,44 11,73 HU IC versus $9,0428,47$ HU CLI $p=0,01)$ No differences were found in the SM quantity $\left(15120,873199,35 \mathrm{~cm}^{2}\right.$ IC versus $15322,575315,31 \mathrm{~cm}^{2}$ CLI $p=0.44$ ).

Conclusion: CLI have a higher quantity of visceral, total AT and lower quantity of subcutaneous AT, SM function and density.

(c) 2019 Association for Research into Arterial Structure and Physiology. Publishing services by Atlantis Press International B.V. This is an open access article distributed under the CC BY-NC 4.0 license (http://creativecommons.org/licenses/by-nc/4.0/). 